\title{
Profile of Evidence-Based Practice Among Respiratory Therapists in Taiwan
}

\author{
Yi-Hao Weng MD, Ken N Kuo MD, Chiehfeng Chen MD, Chun-Yuh Yang PhD, \\ Heng-Lien Lo MA, and Ya-Wen Chiu PhD
}

\begin{abstract}
BACKGROUND: Evidence-based practice (EBP) has been proposed as a core competence to improve healthcare quality. The profile of EBP among respiratory therapists (RTs) has not been explored. We investigated how RTs in Taiwan perceive the implementation of EBP. METHODS: We surveyed RTs in Taiwan's regional hospitals during a 4-month period in 2011. RESULTS: A majority of RTs were aware of EBP $(\mathbf{8 8 . 0 \%})$. Although most RTs held a favorable impression of EBP, their knowledge of and skill in EBP implementation were deficient. Only half of the RTs had implemented EBP. Insufficient convenient kits $(59.1 \%)$, deficient designated personnel $(50.0 \%)$, and lack of time $(45.5 \%)$ were major barriers to implementing EBP. RTs rated MEDLINE as the most commonly used evidence-based retrieval database, followed by UpToDate, the Cochrane Library, MD Consult, ProQuest, CINAHL, DynaMed, and Micromedex. Multivariate regression analyses demonstrated sufficient skill in EBP and use of online databases as favorable factors for implementing EBP. In contrast, barriers of time constraint and insufficient knowledge were unfavorable factors for the implementation of EBP. CONCLUSIONS: EBP is not widespread among RTs in Taiwan. We have identified important factors in the implementation of EBP. The data provide valuable evidence for plotting strategies for disseminating EBP implementation. Key words: evidence-based practice; respiratory therapist; online database. [Respir Care 2014;59(2):281-287. (C) 2014 Daedalus Enterprises]
\end{abstract}

\section{Introduction}

Evidence-based practice (EBP) means clinical practice that is consistent with the current best evidence. EBP integrates clinical epidemiology, biostatistics, research meth-

\footnotetext{
Dr Weng is affiliated with the Division of Neonatology, Department of Pediatrics, Chang Gung Memorial Hospital, Chang Gung University College of Medicine, Taiwan. Dr Kuo is affiliated with the Center for Evidence-Based Medicine, College of Medicine, Taipei Medical University, and with the National Health Research Institutes, Miaoli, Taiwan. Dr Chen is affiliated with the Division of Plastic Surgery, Department of Surgery, and the Evidence-Based Medicine Center, Wan Fang Hospital, and with the Department of Public Health, School of Medicine, and the Center for Evidence-Based Medicine, Taipei Medical University, Taipei, Taiwan. Dr Yang is affiliated with the Department of Public Health, Kaohsiung Medical University, Kaohsiung City, Taiwan. Mr Lo is affiliated with the Center for Evidence-Based Medicine, College of Medicine, Taipei Medical University, Taipei, Taiwan. Dr Chiu is affiliated with the Master Program in Global Health and Development, Health Policy and Care Research Center, College of Public Health and Nutrition, Taipei Medical University, Taipei, Taiwan.
}

ods, and informatics into healthcare. ${ }^{1,2}$ The process of EBP mainly involves 4 steps: framing a clear question based on a clinical problem; finding relevant evidence in the literature; critically appraising the validity of the research; and applying the findings to clinical decision-making. ${ }^{3}$

Respiratory care is increasingly utilized, with the associated respiratory therapists (RTs) playing an important role in supporting related clinical services. ${ }^{4-7}$ EBP has been

\footnotetext{
This study was partly supported by grant PH-099-SP02 from the Taiwan National Health Research Institutes, grant NSC102-2511-S-038-005 from the National Science Council, grant TMU101-AE1-B68 from the Taipei Medical University and grant CMRPG1B0131 from the Chang Gung Memorial Hospital. The authors have disclosed no conflicts of interest.

Correspondence: Ya-Wen Chiu PhD, Master Program in Global Health and Development, College of Public Health and Nutrition, Taipei Medical University, $250 \mathrm{Wu}-\mathrm{H}$ sing Street, Taipei 110, Taiwan. E-mail: bettychiu@tmu.edu.tw.
}

DOI: $10.4187 /$ respcare.02611 
proposed as a useful means to improve healthcare outcomes for RTs. ${ }^{8-11}$ Therefore it is essential to assess how RTs implement EBP in clinical decision-making. A number of studies have illustrated that, although a majority of medical, nursing, pharmacologic, and allied health professions hold positive attitudes toward EBP, their knowledge and skill pertaining to EBP implementation are relatively insufficient. ${ }^{12-18}$ Whether or not RTs practice with evidence, and, if so, how, has been unclear. ${ }^{19}$

RTs have to deal with a broad range of medical problems in clinical practice. ${ }^{20}$ RTs play an important role in the prevention and management of respiratory diseases. ${ }^{21}$ Timely acquired relevant information is important for their clinical practice. Access to evidence-based information can help facilitate effective management. Therefore, how to obtain current evidence-based knowledge is a critical skill. Nevertheless, only a few studies surveyed the behavior of RTs in searching for evidence-based information.

We surveyed RTs in Taiwan regarding their awareness of EBP related to beliefs, attitudes, knowledge, skills, and implementation.

\section{Methods}

The ethical review board of the Taiwan National Health Research Institutes approved the study protocol. The survey was accompanied by an introductory letter stating the purpose of this study and promising confidentiality. Return of the completed questionnaire was considered consent for participation.

\section{Design}

A structured questionnaire was developed by the Taiwan National Health Research Institutes, using questions based on our previously reported questionnaires. ${ }^{13,22}$ This study was conducted in the 4-month period January through April 2011.

\section{Subjects}

We targeted RTs working in Taiwan's regional hospitals. A regional hospital is defined as a secondary-care hospital, as appraised by Taiwan's Joint Commission of Hospital Accreditation. We used cluster sampling: the regional hospitals were divided into 4 clusters (northern, western, eastern, and southern Taiwan), and a random sample of each cluster was selected. Since there are more hospitals in northern and western Taiwan, we selected more hospitals in these areas. We randomly selected 11 of the 65 regional hospitals in Taiwan: 3 in northern Taiwan, 4 in western Taiwan, and 2 each in eastern and southern Taiwan. The questionnaires were distributed by mail to all RTs at the selected hospitals.

\section{QUICK LOOK}

\section{Current knowledge}

Evidence-based medicine drives clinical practice, is a core competency of all members of the health care team, and improves outcomes.

\section{What this paper contributes to our knowledge}

Among respiratory therapists in Taiwan this survey found deficiencies in the knowledge and skill to implement evidence-based practice. The major barriers were lack of time, insufficient materials, and lack of personnel tasked with implementation.

\section{Questionnaire}

The survey used a 5-point Likert scale (strongly agree, agree, neutral, disagree, strongly disagree) for the following questions:

1. Awareness: Have you heard of evidence-based practice (EBP) or related terms, such as evidence-based medicine, evidence-based nursing or evidence-based healthcare?

2. Belief: Do you believe EBP is important for the improvement of patient care quality?

3. Attitude: Are you willing to support the promotion of EBP implementation?

4. Knowledge: Do you have sufficient knowledge to implement EBP principles?

5. Skill: Do you possess enough skill to implement EBP principles?

6. Implementation: Have you searched relevant evidence from the literature to solve your clinical question, and then applied the findings into clinical decision-making after critical appraisal in the past year?

The survey also requested sex, age, faculty position, administrative position, work experience, and academic degree.

The survey used a 5-point Likert scale (always, often, sometimes, seldom, and never) to ask which of the following 8 resources the RTs use to search for professional information: web portals (eg, Google, Yahoo), electronic textbooks, online databases, electronic journals, printed journals, colleague consultations, textbooks, and continuing education (such as conferences).

The survey also asked about the respondents' use of 8 online databases: the Cumulative Index to Nursing and Allied Health Literature (CINAHL), the Cochrane Library, MD Consult, MEDLINE, ProQuest, UpToDate, Micromedex, and DynaMed. These 8 databases were selected because of their popularity. MEDLINE is freely accessi- 
ble, while the other databases require payment from individuals or their organizations. Since 2007 the Taiwan National Health Research Institutes has provided free access to the Cochrane Library for enrolled regional hospitals.

\section{Validity and Reliability}

Content validity was examined by 10 experts, each with more than 15 years of clinical experience. The internal consistency of all the indexes was estimated with the Cronbach alpha coefficient. In this survey the content validity index was 0.96 and the Cronbach alpha coefficient was 0.88 , which indicate sufficient validity and reliability of the parameters in the questionnaire.

\section{Statistical Analyses}

The 5-point Likert scale was dichotomized for further analyses. A self-rating report of either strongly agree or agree was regarded as a favorable answer and the other 3 (neutral, disagree, and strongly disagree) were viewed as unfavorable answers. Similarly, a self-rating report of either always or often was regarded as a favorable answer and the other 3 (sometimes, seldom, and never) were viewed as unfavorable answers. Analyses were conducted with statistics software (SPSS 12.0, SPSS, Chicago, Illinois). Categorical variables were analyzed using the chi-square or Fisher exact test. Significance was defined as $P<.05$.

\section{Results}

A total of 86 questionnaires were distributed to RTs, and 50 returned surveys were valid for analysis (valid return rate $58.1 \%$, Table 1 ). The average age and working period were 32.8 years \pm 6.0 years and 4.8 years \pm 4.7 years, respectively. Three RTs had a master's degree (6.0\%), while 34 had a bachelor's degree (68.0\%), 10 had a degree from a junior college (20.0\%), and the rest had a degree from a technical school $(6.0 \%)$. Furthermore, 13 RTs were faculty members $(26.0 \%)$ and 5 were directors $(10.0 \%)$.

\section{Awareness, Beliefs, Attitudes, Knowledge, Skills, and Training Related to EBP}

Forty-four RTs (88.0\%) were aware of EBP. Their belief in, attitude toward, knowledge of, and skill in EBP are shown in Figure 1. Thirty-eight RTs believed that EBP is important for the improvement of patient-care quality (86.4\%). In addition, 27 RTs stated that they were willing to support the implementation of EBP (61.4\%). However, their knowledge of implementing EBP principles (29.5\%)
Table 1. Demographics of the 50 Respondent Respiratory Therapists

\begin{tabular}{lrr}
\hline \hline & No. & $\%$ \\
\hline Male & 5 & 10 \\
Female & 45 & 90 \\
Age range, y & & \\
$20-30$ & 16 & 32 \\
$31-40$ & 28 & 56 \\
$41-50$ & 6 & 12 \\
Work experience range, y & & \\
$<5$ & 31 & 62 \\
$5-10$ & 15 & 30 \\
$>10$ & 4 & 8 \\
Academic degree & & 6 \\
Technical school & 3 & 6 \\
Junior college & 10 & \\
Bachelor's & 34 & 26 \\
Master's & 3 & 74 \\
Faculty member, \% & & \\
Yes & 13 & 90 \\
$\quad$ No & 37 & \\
Director, \% & & \\
Yes & 5 & \\
No & 45 & \\
\hline
\end{tabular}

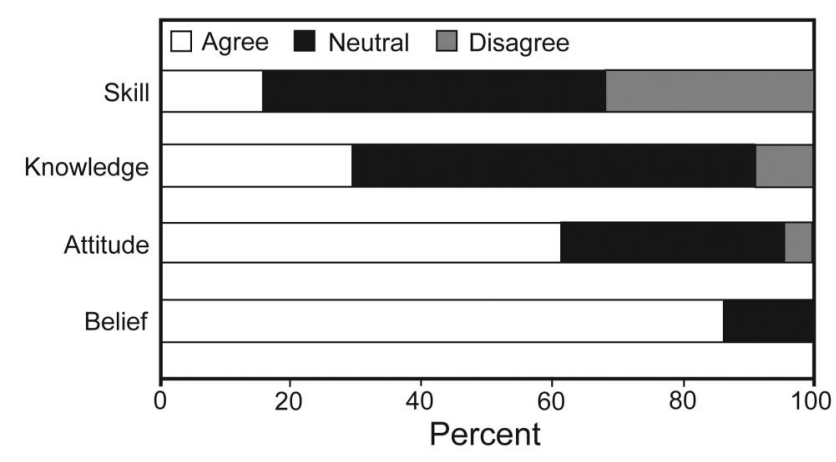

Fig. 1. Belief in, attitude toward, knowledge of, and skill in evidence-based practice among 44 respiratory therapists in regional hospitals in Taiwan.

and skill in implementing EBP principles (15.9\%) were relatively insufficient. Of the 44 RTs who were aware of EBP, 11 RTs $(25.0 \%)$ had participated in a training course for EBP implementation.

\section{Barriers to Implementing EBP}

The barriers to implementing EBP are illustrated in Figure 2. The most common barrier was insufficient convenient kits (such as personal digital assistants, software, sketches, diagrams, fly sheets, manuals, guidebooks, molds, and brochures) (59.1\%). Common barriers included deficient capable designated personnel (50.0\%), time constraints $(45.5 \%)$, deficient skill in critical appraisal 


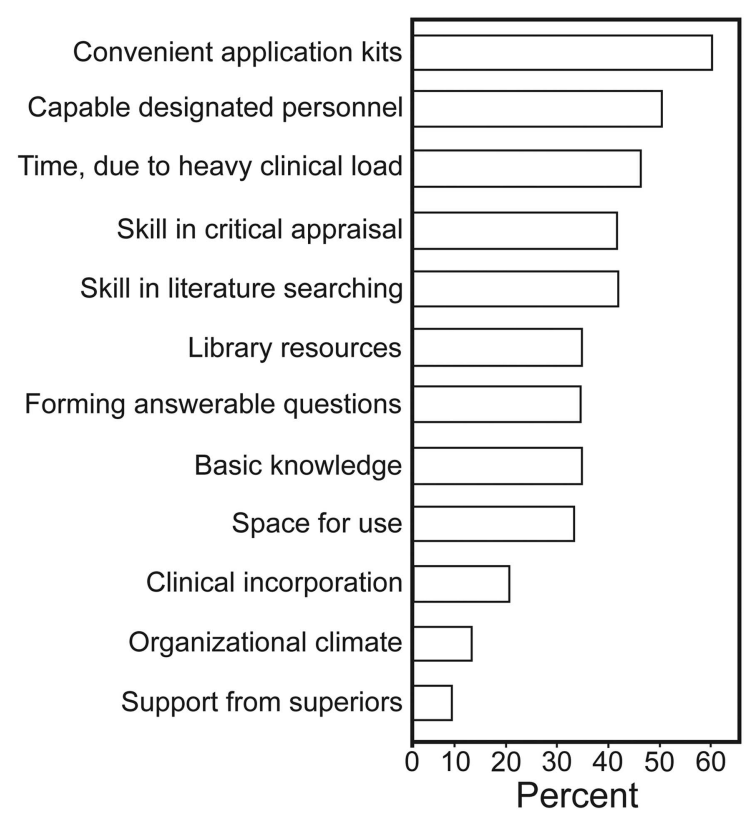

Fig. 2. Perceived barriers to the implementation of evidence-based practice.

(40.9\%), deficient skill in literature searching (40.9\%), insufficient library resources $(34.1 \%)$, difficulty in forming answerable questions (34.1\%), deficient basic knowledge $(34.1 \%)$, deficient space for use $(31.8 \%)$, lack of incorporation with clinical practice $(20.5 \%)$, lack of organizational climate (13.6\%), and lack of support from superiors $(9.1 \%)$.

\section{Implementation of EBP}

Among the 44 RTs who were aware of EBP, 25 reported that they had implemented EBP in the past year (56.8\%), including 2 RTs who had implemented EBP daily (4.6\%), 1 weekly (2.3\%), 4 monthly (9.1\%), 9 quarterly (20.5\%), and 9 yearly $(20.5 \%)$. The remaining 19 RTs had not implemented EBP for clinical decision-making in the past year $(43.2 \%)$.

\section{Search for Evidence-Based Information}

The 50 RTs' information-searching behavior is shown in Figure 3. Of the 8 resources, RTs most often searched for professional information via Web portals (84.0\%), followed by colleague consultations $(72.0 \%)$, continuing education $(66.0 \%)$, evidence-based retrieval databases $(58.0 \%)$, textbooks $(56.0 \%)$, electronic journals $(46.0 \%)$, electronic books $(28.0 \%)$, and printed journals $(22.0 \%)$.

The frequency of access to 8 commonly used online evidence-based retrieval databases is given in Figure 4.

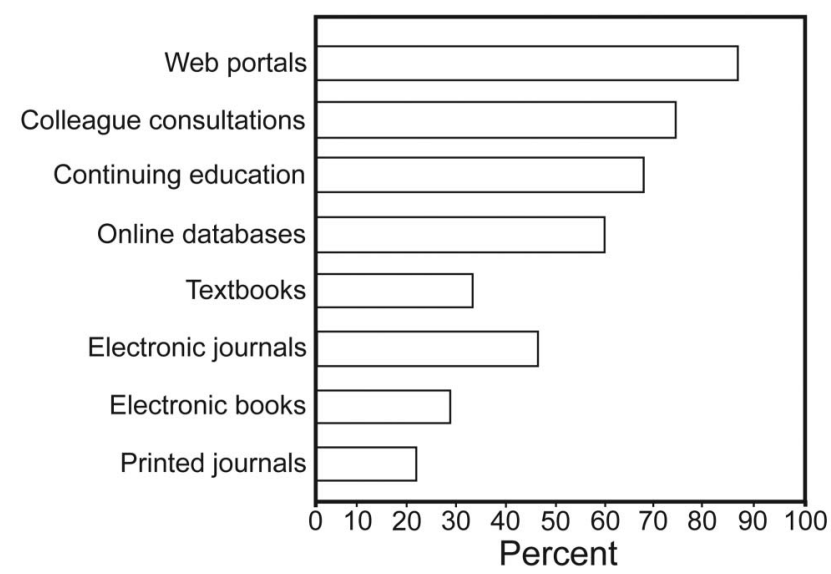

Fig. 3. Behavior of searching for professional information.

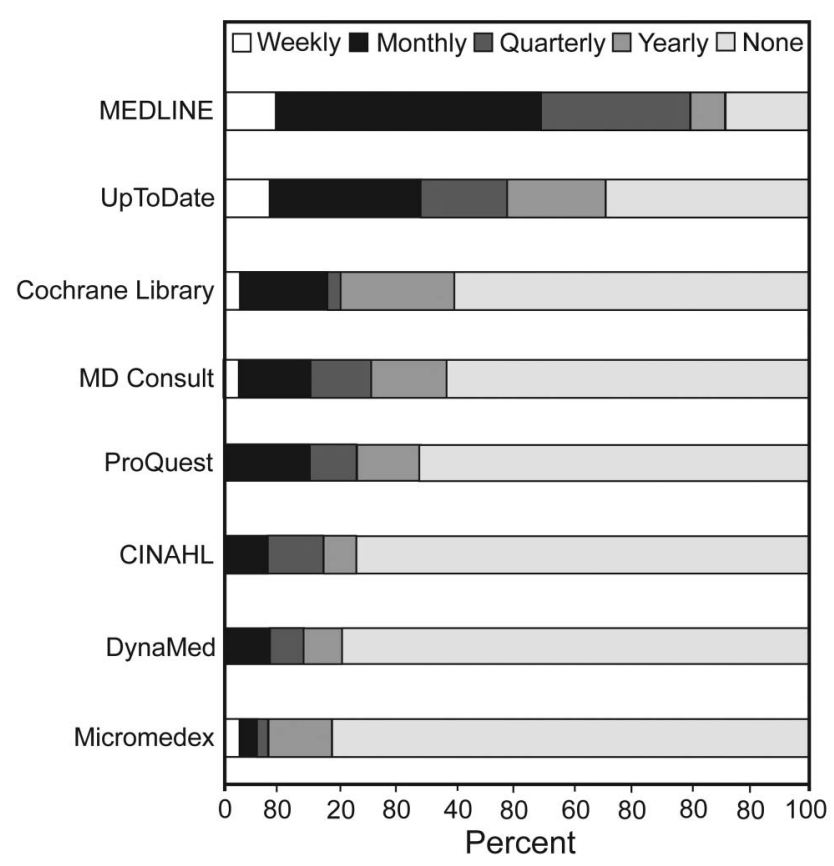

Fig. 4. Frequency of access to 8 commonly used online evidencebased retrieval databases.

The most commonly used database was MEDLINE, followed by UpToDate, the Cochrane Library, MD Consult, ProQuest, CINAHL, DynaMed, and Micromedex.

Among the 44 RTs who were aware of EBP, 29 had accessed online evidence-based retrieval databases. Their motivations for accessing such databases are shown in Table 2. The most common motivation was self-learning (100\%), followed by clinical practice $(89.7 \%)$, class assignment (72.4\%), positional promotion (62.1\%), instruction preparation $(48.3 \%)$, research $(37.9 \%)$, medical accreditation (31.0\%), and insurance issue $(6.9 \%)$. 
Table 2. Motivations for Accessing the Evidence-Based Retrieval Database Among Taiwanese Respiratory Therapists

\begin{tabular}{llcc}
\hline \hline Rank & \multicolumn{1}{c}{ Motivation } & No. & $\%$ \\
\hline 1 & Self-learning & 29 & 100 \\
2 & Clinical practice & 26 & 89.7 \\
3 & Class assignment & 21 & 72.4 \\
4 & Positional promotion & 18 & 62.1 \\
5 & Instruction preparation & 14 & 48.3 \\
6 & Research & 11 & 37.9 \\
7 & Medical accreditation & 9 & 31.0 \\
8 & Insurance issue & 2 & 6.9 \\
\hline
\end{tabular}

\section{Factors Associated With EBP Implementation}

Factors in relation to the implementation of EBP are shown in Table 3. Compared with RTs without EBP implementation in clinical practice, RTs with EBP implementation were more likely to have sufficient skill in EBP $(P=.01)$ and more often have accessed relevant online evidence-based retrieval databases $(P=.046)$. In addition, RTs who listed time constraint $(P=.040)$ and lack of basic knowledge $(P=.02)$ as barriers were less likely to implement EBP than those who did not perceive these barriers. The behavior of information searching and demographic characteristics, including sex, position, working experience, age, and academic degree, carried no significant difference.

\section{Discussion}

A majority of Taiwanese RT survey respondents had a favorable perception of EBP. However, their knowledge of EBP and skill pertaining to implementing it in clinical decision-making were relatively insufficient. The findings are in accordance with reports about physicians, nurses, and the other allied health professionals. ${ }^{12-18}$ To our knowledge, this study is the first to explore the EBP profile of registered RTs.

There have been numerous efforts to determine the barriers to implementing EBP. ${ }^{13,23}$ Our results indicate that RTs encounter a number of impedances. Overall, lack of convenient kits and insufficient numbers of capable designated personnel were the most common barriers. Unlike medical centers, most regional hospitals are understaffed because of budget restraints. Since EBP is to a large extent a working framework, RTs need to find help from designated staff to overcome obstacles. Like the other health professionals, RTs lack sufficient time to search information in a pile of textbooks or journals. Thus, they require useful information that they can acquire at their own convenience. With the help of application kits and capable designated personnel, they could save time and overcome
Table 3. Factors Related to Implementation of Evidence-Based Practice

\begin{tabular}{|c|c|c|c|}
\hline & $\begin{array}{c}\text { Yes } \\
(n=25)\end{array}$ & $\begin{array}{c}\text { No } \\
(n=19)\end{array}$ & $P$ \\
\hline Belief & $22(88.0)$ & $16(84.2)$ & $>.99$ \\
\hline Attitude & $17(68.0)$ & $10(52.6)$ & .30 \\
\hline Knowledge & $8(32.0)$ & $5(26.3)$ & .68 \\
\hline Skill & $7(28.0)$ & $0(0.0)$ & .01 \\
\hline Training & $9(36.0)$ & $2(10.5)$ & .08 \\
\hline \multicolumn{4}{|l|}{ Barrier/difficulty } \\
\hline Convenient application kits & $16(64.0)$ & $10(52.6)$ & .45 \\
\hline Capable designated personnel & $13(52.0)$ & $9(47.4)$ & .76 \\
\hline Time, due to heavy clinical load & $8(32.0)$ & $12(63.2)$ & .040 \\
\hline Skill in critical appraisal & $8(32.0)$ & $10(52.6)$ & .17 \\
\hline Skill in literature searching & $8(32.0)$ & $10(52.6)$ & .17 \\
\hline Library resources & $7(28.0)$ & $8(42.1)$ & .33 \\
\hline Forming answerable questions & $6(24.0)$ & $9(47.4)$ & .11 \\
\hline Basic knowledge & $5(20.0)$ & $10(52.6)$ & .02 \\
\hline Space for use & $8(32.0)$ & $6(31.6)$ & .98 \\
\hline Clinical incorporation & $3(12.0)$ & $6(31.6)$ & .14 \\
\hline Organizational climate & $5(20.0)$ & $1(5.3)$ & .21 \\
\hline Support from superiors & $3(12.0)$ & $1(5.3)$ & .62 \\
\hline \multicolumn{4}{|l|}{ Information searching } \\
\hline Skill in literature searching & $22(88.0)$ & $10(52.6)$ & .009 \\
\hline \multicolumn{4}{|l|}{ Resource of information } \\
\hline Web portals & $22(88.0)$ & $15(78.9)$ & .44 \\
\hline Colleague consultations & $18(72.0)$ & $13(68.4)$ & .80 \\
\hline Continuing education & $19(76.0)$ & $11(57.9)$ & .20 \\
\hline Online databases & $18(72.0)$ & $8(42.1)$ & .046 \\
\hline Textbooks & $12(48.0)$ & $11(57.9)$ & .52 \\
\hline Electronic journals & $13(52.0)$ & $7(36.8)$ & .32 \\
\hline Electronic books & $6(24.0)$ & $6(31.6)$ & .58 \\
\hline Printed journals & $4(16.0)$ & $5(26.3)$ & .47 \\
\hline \multicolumn{4}{|l|}{ Demographic characteristics } \\
\hline Female & $24(96.0)$ & $17(89.5)$ & .57 \\
\hline Director & $4(16.0)$ & $4(5.3)$ & .37 \\
\hline Faculty & $8(32.0)$ & $5(26.3)$ & .68 \\
\hline Work experience range, $\mathrm{y}$ & & & .98 \\
\hline$<5$ & $14(56.0)$ & $11(57.9)$ & \\
\hline $5-10$ & $9(36.0)$ & $6(31.6)$ & \\
\hline$>10$ & $2(8.0)$ & $2(10.5)$ & \\
\hline Age range, y & & & .40 \\
\hline $20-30$ & $6(24.0)$ & $8(42.1)$ & \\
\hline $31-40$ & $17(68.0)$ & $9(47.4)$ & \\
\hline $41-50$ & $2(8.0)$ & $2(10.5)$ & \\
\hline Academic degree & & & .67 \\
\hline Technical school & $2(8.0)$ & $1(5.3)$ & \\
\hline Junior college & $3(12.0)$ & $5(26.2)$ & \\
\hline Bachelor's & $18(72.0)$ & $12(63.2)$ & \\
\hline Master's & $2(8.0)$ & $1(5.3)$ & \\
\hline
\end{tabular}

the constraints of insufficient knowledge and skill. In addition, RTs rated insufficient knowledge and skill as significant barriers to the practice with evidence. Only a 
minority of RTs had received EBP-related training. These findings can provide information for policymakers to plot strategies to disseminate the implementation of EBP for RTs. For example, education to enhance RTs' knowledge of EBP and their skill in its implementation may be helpful.

Our study has verified several factors in relation to the implementation of EBP. First, self-efficacy in the skill of EBP is the primary influence on its implementation. The finding is similar to previous reports indicating that health professionals who often implemented EBP had more sufficient skill. ${ }^{24-27}$ Second, lacking knowledge and time serve as 2 negative predictors. Taken together, our data support the importance of providing training courses to facilitate the implementation of EBP. The data concur with other available studies showing that teaching programs can change the behavior of EBP implementation. ${ }^{3,28}$

The respondent RTs use a wide variety of approaches to look for professional information. We found that Web portals were the most popular resource, possibly because access to Web portals is instantaneous. However, information found via Web portals is not always accurate. On the other hand, online databases can offer evidence-based and summarized recommendations for clinical services to facilitate the integration of evidence into practice. ${ }^{29} \mathrm{~A}$ high proportion of the respondent RTs used online databases for self-learning and clinical service. They accessed MEDLINE the most, which is not surprising because it provides more information than the other databases. Our study is the first to identify the patterns of RTs' preferences in information searching. The data can help refine strategies to promote the accessing of evidence-based information.

When compared with physicians in Taiwan, ${ }^{13}$ the knowledge and skills of EBP among Taiwanese RTs is relatively low. The likely explanation is that physicians have been persuaded to follow EBP longer than RTs have. In Taiwan the respiratory therapy profession is still in the developing stage. In the past, RTs relied mainly on clear direction from physicians rather than their own decisionmaking. The regulatory standards for clinical practice and the process for attaining credentials were not well established until recently.

There are limitations to our study. First, this was a self-administered survey: not an audit of actual practice. The results may not reflect the realities of practice under routine clinical care. Second, the survey-return rate was only $58.1 \%$, though that rate is similar to other RTs surveys in Taiwan on different subjects. ${ }^{30}$ To optimize the response rate we used a cluster sampling, which improves response rate, compared to random sampling. ${ }^{31}$ Third, our sample size was small. Nevertheless, our study is the pioneer in evaluating the EBP profile of RTs. Despite these limitations, our survey presents several potentially useful findings. Our study differs from previous ones on information-searching patterns, in that we examined the EBP-related behaviors in the context of clinical decisionmaking.

\section{Conclusions}

This cross-sectional survey provides baseline data about the perceptions of EBP among registered RTs in regional hospitals in Taiwan. Although RTs recognize the value of EBP, few have acquired the ability to implement its principles. Thus, RTs in Taiwan are not ready for EBP, because of insufficient knowledge and skills. The findings have important implication for educational and clinical issues to disseminate the EBP implementation into RTs. We suggest that RTs in the regional hospitals of Taiwan require more EBP-related training courses to improve their knowledge and skills.

\section{ACKNOWLEDGMENTS}

We are grateful to the respondents who gave their time in this study. We thank Ya-Hui Shih for her dedicated work on the collection of research data.

\section{REFERENCES}

1. Cook DJ. Moving toward evidence-based practice. Respir Care 2003; 48(9):859-868.

2. Weng YH, Hsu CC, Shih YH, Lo HL, Chiu YW, Kuo KN. Dissemination of systematic reviews in a hospital setting: a comparative survey for spreading use of the Cochrane Library. Postgrad Med J 2012;88(1043):511-514.

3. Thomas A, Saroyan A, Dauphinee WD. Evidence-based practice: a review of theoretical assumptions and effectiveness of teaching and assessment interventions in health professions. Adv Health Sci Educ Theory Pract 2011;16(2):253-276.

4. Colice GL, Carnathan B, Sung J, Paramore LC. A respiratory therapist-directed protocol for managing inpatients with asthma and COPD incorporating a long-acting bronchodilator. J Asthma 2005; 42(1):29-34.

5. Stoller JK, Skibinski CI, Giles DK, Kester EL, Haney DJ. Physicianordered respiratory care vs physician-ordered use of a respiratory therapy consult service. Results of a prospective observational study. Chest 1996;110(2):422-429.

6. Kareus SA, Kagebein S, Rudnicki SA. The importance of a respiratory therapist in the ALS clinic. Amyotroph Lateral Scler 2008; 9(3):173-176.

7. Kallstrom TJ, Myers TR. Asthma disease management and the respiratory therapist. Respir Care 2008;53(6):770-776.

8. Tramacere A, Rizzardi R, Cilione C, Serri B, Florini F, Lorenzi MC, et al. Effects of respiratory therapist-directed protocol on prescription and outcomes of pulmonary rehabilitation in COPD inpatients. Respiration 2004;71(1):60-65.

9. Harbrecht BG, Delgado E, Tuttle RP, Cohen-Melamed MH, Saul MI, Valenta CA. Improved outcomes with routine respiratory therapist evaluation of non-intensive-care-unit surgery patients. Respir Care 2009;54(7):861-867.

10. Hermeto F, Bottino MN, Vaillancourt K, Sant'Anna GM. Implementation of a respiratory therapist-driven protocol for neonatal ventilation: impact on the premature population. Pediatrics 2009;123(5): e907-916. 


\section{Profile of Evidence-Based Practice Among Respiratory Therapists in Taiwan}

11. Stoller JK, Hoisington ER, Lemin ME, Karol JA, Chatburn RL, Mascha EJ, et al. Concordance of respiratory care plans generated by protocols from different hospitals: a comparative study. Respir Care 2007;52(8):1006-1012.

12. Chiu YW, Weng YH, Wahlqvist ML, Yang CY, Kuo KN. Do registered dietitians search for evidence-based information? A nationwide survey of regional hospitals in Taiwan. Asia Pac J Clin Nutr 2012;21(4):630-637.

13. Chiu YW, Weng YH, Lo HL, Hsu CC, Shih YH, Kuo KN. Comparison of evidence-based practice between physicians and nurses: a national survey of regional hospitals in Taiwan. J Contin Educ Health Prof 2010;30(2):132-138

14. Burkiewicz JS, Zgarrick DP. Evidence-based practice by pharmacists: utilization and barriers. Ann Pharmacother 2005;39(7-8):1214-1219.

15. Heiwe S, Kajermo KN, Tyni-Lenne R, Guidetti S, Samuelsson M, Andersson IL, et al. Evidence-based practice: attitudes, knowledge and behaviour among allied health care professionals. Int J Qual Health Care 2011;23(2):198-209.

16. Jette DU, Bacon K, Batty C, et al. Evidence-based practice: beliefs, attitudes, knowledge, and behaviors of physical therapists. Phys Ther 2003;83(9):786-805.

17. Knops AM, Vermeulen H, Legemate DA, Ubbink DT. Attitudes, awareness, and barriers regarding evidence-based surgery among surgeons and surgical nurses. World J Surg 2009;33(7):1348-1355.

18. Lai NM, Teng CL, Lee ML. The place and barriers of evidence based practice: knowledge and perceptions of medical, nursing and allied health practitioners in malaysia. BMC Res Notes 2010;3:279.

19. Stelfox HT, Hess DR, Schmidt UH. A North American survey of respiratory therapist and physician tracheostomy decannulation practices. Respir Care 2009;54(12):1658-1664.

20. Prewitt MW. Role expectations of the respiratory therapy practitioner as reported by the role incumbent and other health care professionals. Respir Care 1984;29(9):900-904.

21. Sandrock C, Daly J. The importance of a multidisciplinary approach to VAP prevention: the role of the respiratory therapist. Respir Care 2012;57(5):811-812.
22. Chiu YW, Weng YH, Lo HL, Shih YH, Hsu CC, Kuo KN. Comparison of accessing online databases between physicians and nurses in Taiwan. Inform Health Soc Care 2012;37(4):230-241.

23. Ely JW, Osheroff JA, Ebell MH, et al. Obstacles to answering doctors' questions about patient care with evidence: qualitative study. BMJ 2002;324(7339):710.

24. Squires JE, Estabrooks CA, Gustavsson P, Wallin L. Individual determinants of research utilization by nurses: a systematic review update. Implement Sci 2011;6:1.

25. Skoglund I, Segesten K, Bjorkelund C. GPs' thoughts on prescribing medication and evidence-based knowledge: the benefit aspect is a strong motivator. A descriptive focus group study. Scand J Prim Health Care 2007;25(2):98-104.

26. McAlister FA, Graham I, Karr GW, Laupacis A. Evidence-based medicine and the practicing clinician. J Gen Intern Med 1999;14(4): 236-242.

27. Bostrom AM, Kajermo KN, Nordstrom G, Wallin L. Barriers to research utilization and research use among registered nurses working in the care of older people: does the BARRIERS Scale discriminate between research users and non-research users on perceptions of barriers? Implement Sci 2008;3:24.

28. Straus SE, Ball C, Balcombe N, Sheldon J, McAlister FA. Teaching evidence-based medicine skills can change practice in a community hospital. J Gen Intern Med 2005;20(4):340-343.

29. Chiu YW, Weng YH, Lo HL, et al. Physicians' characteristics in the usage of online database: a representative nationwide survey of regional hospitals in Taiwan. Inform Health Soc Care 2009;34(3): 127-135.

30. Shie HG, Lee WC, Hsiao HF, Lin HL, Yang LL, Jung F. Patient safety attitudes among respiratory therapists in Taiwan. Respir Care 2011;56(12):1924-1929.

31. Chiu YW, Weng YH, Lo HL, Hsu CC, Shih YH, Kuo KN. Impact of a nationwide outreach program on the diffusion of evidence-based practice in Taiwan. Int J Qual Health Care 2010; 22(5):430-436 